\title{
Gastric tuberculosis resembling depressed type,
} early gastric cancer

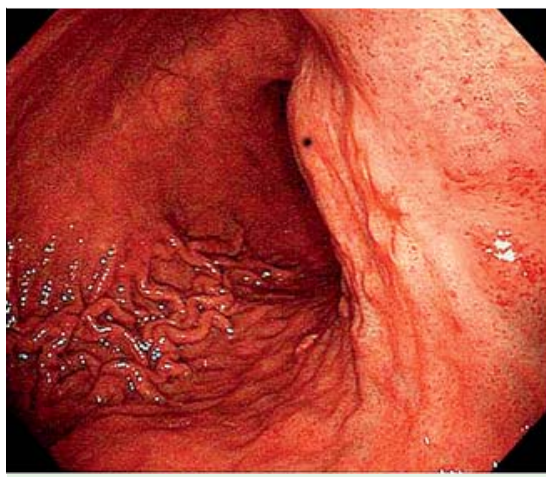

Fig. 1 Esophagogastroduodenoscopy (EGD) showing a depressed lesion with converging folds on the posterior wall of the upper gastric corpus in a 60-year-old asymptomatic man.

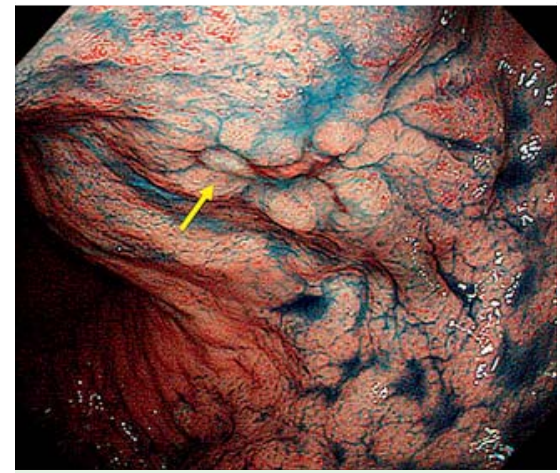

Fig. 2 EGD with chromoendoscopy revealed an irregularly shaped, depressed lesion of which the anal portion appeared to resemble a whitish nodule (arrow).

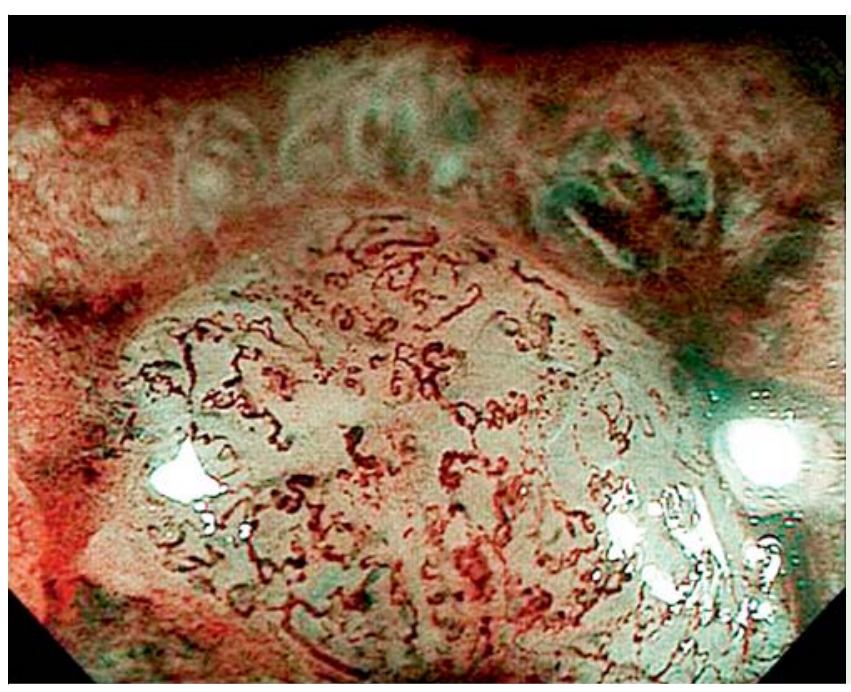

Fig. 3 Image enhanced endoscopy with magnifying narrow-band imaging demonstrating the spiral-shaped, dilated small vessels or microvessels with an amorphous surface.

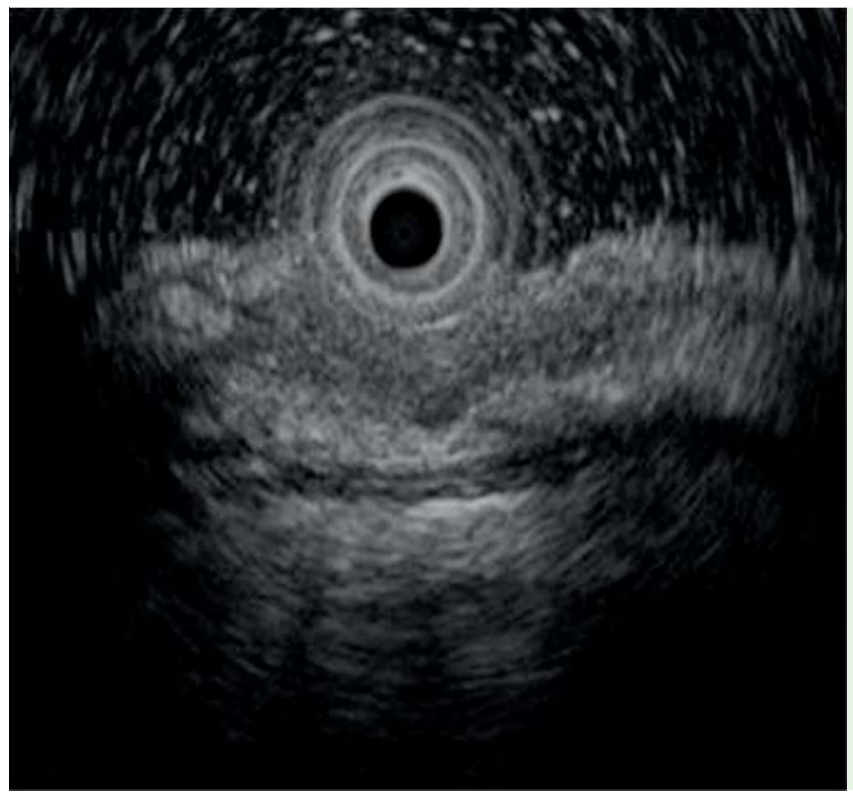

Fig. 4 Endoscopic ultrasonography (EUS) demonstrating a hypoechoic lesion localized in the deep portion of the mucosa and the superficial submucosa.
A 60-year-old asymptomatic man was referred to our hospital for evaluation of a gastric lesion detected by esophagogastroduodenoscopy (EGD) in a medical check-up. EGD showed an irregularly shaped, depressed lesion with converging folds on the posterior wall of the upper gastric corpus ( Fig. 1, Fig.2). Magnifying endoscopy with narrow-band imaging at the anal portion of the lesion revealed spiral-shaped, dilated small vessels/microvessels with an amorphous surface ( Fig.3). Endoscopic ultrasonography (EUS) demonstrated a hypoechoic lesion localized in the deep portion of the mucosa and the superficial submucosa ( Fig. 4). A biopsy from the lesion showed granulomatous inflammation with caseous necrosis and Langerhans giant cells. Acid-fast bacilli were detected by both Ziehl-Neelsen staining ( $\bullet$ Fig.5) and mycobacterium culture. A polymerase chain reaction test for tuberculosis was also positive. Fluorine-18 fluorodeoxyglucose positron emission tomography (PET) showed markedly increased accumulation in the lymph nodes of the mediastinum, pulmonary hilum, and upper abdomen ( Fig.6). Chest computed tomography (CT) revealed no evidence of pulmonary tuberculosis. Colonoscopy, small-bowel capsule endoscopy, and bronchoscopy showed normal findings. Biopsy from the inguinal lymph node demonstrated nonspecific inflammation without any neoplastic cells or granulomas. Thus, the patient was diagnosed as having gastric tuberculosis with systemic lymphadenopathy, and subsequently underwent antituberculous treatment. Both the gastric lesion and lymphadenopathy had resolved 6 months later.

Gastric tuberculosis is rare, and its endoscopic appearance can vary [1 -4]. To confirm a definitive diagnosis of tuberculosis, EUS-guided, fine-needle aspiration or surgery is sometimes required $[1,4,5]$. The gastric lesion in our case resembled depressed-type, early gastric cancer endoscopically; however when the amorphous area (probably composed of inflammation or granulation tissue covered with thin epithelium) was viewed using magnifying endoscopy with narrow-band imaging, it was clearly different from that of gastric cancer. We thus consider that magnifying endoscopy with narrow-band imaging is useful in the differential diagnosis between gastric tuberculosis and gastric cancer. 

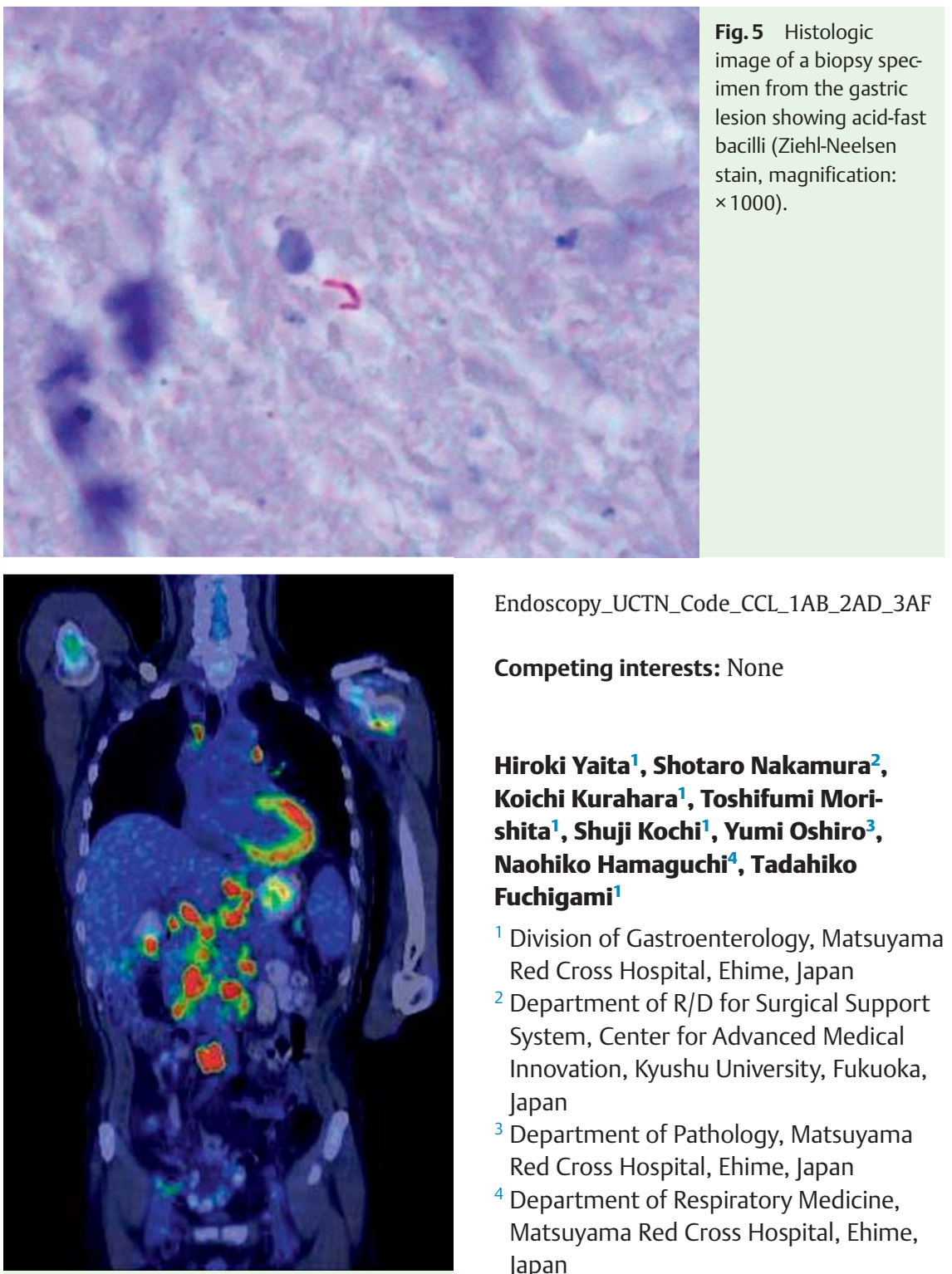

Fig. 6 Fluorine-18 fluorodeoxyglucose positron emission tomography (PET) showing markedly increased accumulation in the lymph nodes of the mediastinum, pulmonary hilum, and upper abdomen. Fuchigami ${ }^{1}$

\section{References}

1 Liu PF, Chang CS, Wang J et al. Primary gastric tuberculosis. Endoscopy 2009; 41: E327E328

2 Lim JU, Kim YH, Choi CW et al. Gastric tuberculosis presenting with a huge abdominal mass. Singapore Med J 2013; 54: e244e246

3 Kim DY, Bang S, Park BK et al. Tuberculous mesenteric lymphadenitis involving the gastric wall: case report. Gastrointest Endosc 2005; 62: 799-802

$4 \mathrm{Kim} \mathrm{SH}$, ParkJH, Kang KH et al. Gastric tuberculosis presenting as a submucosal tumor. Gastrointest Endosc 2005; 61: 319-322

5 Rana SS, Bhasin DK, Srinivasan R et al. Gastric outlet obstruction caused by tuberculosis and diagnosed by endoscopic ultrasound-guided fine needle aspiration. Endoscopy 2011; 43: E117-E118

\section{Bibliography}

DOI http://dx.doi.org/

10.1055/s-0034-1390865

Endoscopy 2014; 46: E669-E670

(c) Georg Thieme Verlag KG

Stuttgart · New York

ISSN 0013-726X

Hiroki Yaita', Shotaro Nakamura²,

shita $^{1}$, Shuji Kochi ${ }^{1}$, Yumi Oshiro ${ }^{3}$, Naohiko Hamaguchi ${ }^{4}$, Tadahiko

${ }^{1}$ Division of Gastroenterology, Matsuyama Red Cross Hospital, Ehime, Japan

${ }^{2}$ Department of R/D for Surgical Support System, Center for Advanced Medical Innovation, Kyushu University, Fukuoka, Japan

${ }^{3}$ Department of Pathology, Matsuyama

Red Cross Hospital, Ehime, Japan

${ }^{4}$ Department of Respiratory Medicine, Matsuyama Red Cross Hospital, Ehime, Japan
Corresponding author

\section{Hiroki Yaita, MD}

Division of Gastroenterology

Matsuyama Red Cross Hospital

1 Bunkyo-cho

Matsuyama-shi

Ehime 790-8524

Japan

Fax: +81-89-9269916

hyaita@matsuyama.jrc.or.jp 